\title{
HACIA LA CONVERGENCIA DE LOS PROCESOS DE INTEGRACIÓN EN AMÉRICA DEL SUR
}

\section{TOWARDS THE CONVERGENCE OF INTEGRATION PROCESSES IN SOUTH AMERICA}

\author{
Renzo ChIrI MÁrqueZ*
}

Recibido: 12/06/2017

Aceptado: 10/08/2017

\section{Resumen}

El presente trabajo analiza la actualidad de los diversos procesos de integración existentes

* Abogado graduado en la Universidad de Lima (1994). Egresado de la Maestría en Gestión Pública en EUCIM Bussines School y el Instituto de Gobierno y Gestión Pública de la Universidad de San Martín de Porres. Master en Gerencia Pública por EUCIM Bussines School (2016). Postgrado de Especialización en Derecho Constitucional y Ciencia Política en el Centro de Estudios Constitucionales de Madrid, España (1995-1996). Estudios de "Planificación y Gestión de Recursos para la Defensa” (2003) y "Cooperación Interinstitucional y Contraterrorismo" (2005) en el Centro de Estudios Hemisféricos de Defensa (CHDS) en Washington D.C. Es graduado del Programa de Especialización en Gestión Estratégica del Sector Público de la Universidad del Pacífico. Fue Comisionado del Defensor del Pueblo (1996-2000) y Secretario General de la Comisión Andina de Juristas (2001-2006). También fue Secretario General del Ministerio de Defensa del Perú entre agosto del 2006 y enero del 2011, Asesor en la Comisión Nacional para el Desarrollo y Vida sin Drogas-DEVIDA (2012) y Oficial del Programa de Libertades Informativas del Instituto Prensa y Sociedad - IPYS (2012-2013). Actualmente es asesor en temas de Gestión Pública en la Gerencia Legal de OSIPTEL. Profesor de los cursos de Derechos Humanos, Teoría del Estado y Derecho de la Integración en la Facultad de Derecho y Ciencia Política de la Universidad Ricardo Palma. en América del Sur: sus diversas orientaciones y énfasis, sus denominadores comunes y sus divergencias, pero sobre todo, la necesidad de impulsar esfuerzos y sinergias buscando la convergencia de los mismos en un gran proyecto de integración subregional. La convergencia institucional de los diversos procesos de integración puestos en marcha en América del Sur, puede servir como un instrumento eficaz para alcanzar mayores niveles de bienestar económico y social, para la profundización y el mejoramiento de los estándares democráticos en la región, y para forjar un bloque geopolítico que nos permita convertir a Sudamérica en un actor internacional que aproveche y gestione mejor las oportunidades que genera la globalización.

\section{Abstract}

This paper analyzes the current estate of the diverse integration processes in South America; their different orientations and emphasis, their common denominators and divergences, but mainly, their necessity to impulse efforts and synergies searching for their convergence in a great sub regional integration project. The institutional convergence of the diverse 
integration processes set in motion in South America, may serve as an efficient instrument to achieve greater levels of economic and social well-being, deepening and improvement of the regional democratic standards and forge a geopolitical block that allows us to transform South America in to an international actor that takes advantage and manages the opportunities generated by globalization better.

\section{Palabras Clave}

Procesos de Integración - América del Sur - Convergencia - Instituciones - Subregión Globalización - Comunidad Andina - Mercado Común del Sur - Unión de Naciones Suramericanas - Alianza del Pacífico - Asociación Latinoamericana de Integración.

\section{Key Words}

Integration processes - South America Convergence - Institutions - Subregión - Globalization - Andean Community - Southern Common Market - Union of South American Nations - Pacific Alliance - Latin American Integratión Association.

\section{El camino de la Integración.-}

Tras el final de la II Guerra Mundial, se han producido diversas iniciativas orientadas a crear bloques de Estados con fines de integración económica, política y social. Esta etapa de creación de procesos de integración, tiene sus primeros capítulos con la aparición de la Comunidad Económica del Carbón y el Acero (CECA) ${ }^{1}$, la Agencia Europea de Energía Atómica (EURATOM) y la Comunidad Económica Europea $(\mathrm{CEE})^{2}$, todas ellas insti-

1 Creada a través de la suscripción del Tratado de París de 1951.

2 Ambas instituciones fueron creadas a partir de la suscripción de los Tratados de Roma de 1957. tuciones que constituyeron los primeros pasos del proceso de integración europeo que ha dado como resultado a la moderna Unión Europea de hoy.

Nuestra América Latina no ha sido ajena a esta corriente. Es así que, desde los años sesenta del siglo XX, encontramos procesos de integración tan diversos como la Asociación Latinoamericana de Libre Comercio (ALALC) ${ }^{3}$, la Organización de Estados Centroamericanos (ODECA $)^{4}$, la Asociación Caribeña de Libre Cambio ${ }^{5}$ y el Acuerdo de Cartagena ${ }^{6}$. Ya en el inicio del siglo XXI, la preocupación por la integración regional se mantiene vigente y se ha expresado en la creación de procesos de la envergadura y proyección de la Unión de Naciones Suramericana - UNASUR $(2004)^{7}$ y la Alianza del Pacífico (2011) ${ }^{8}$.

\section{El Horizonte de la Integración Sudameri- cana.-}

Desde nuestra perspectiva, la pregunta ¿nos conviene apostar por la integración de América del Sur?, sólo puede ser respondida con un SI claro y rotundo. Avanzar decidida-

3 El ALALC, antecesor de la actual ALADI, fue creado a través de la suscripción del Tratado de Montevideo de 1960.

4 La ODECA, antecesor del actual Sistema de Integración Centroamericano (SICA), se creó con la firma del Protocolo de San Salvador de 1962.

5 La Asociación Caribeña de Libre Cambio, antecesor de la actual Comunidad del Caribe (CARICOM), fue creada en 1965 .

6 El Acuerdo de Cartagena, nombre original de la actual Comunidad Andina, nace con la suscripción del tratado del mismo nombre en Cartagena de Indias, Colombia, en 1969.

7 La UNASUR, creada originalmente como Comunidad Sudamericana de Naciones, nace en el Cuzco en diciembre de 2004 durante el desarrollo de una Reunión de Presidentes de América del Sur.

8 Iniciativa creada por Chile, Colombia, México y el Perú en abril del 2011 a través de la Declaración de Lima. 
mente hacia el logro de este objetivo, no debe verse nunca de un modo idílico o romántico, sino todo lo contrario. Trabajar arduamente para consolidar progresivamente un proceso de integración sudamericano, debe considerarse como un objetivo político-estratégico de máxima prioridad, basado en razones pragmáticas y de absoluta conveniencia económica, política y social para todos los países que integran ésta subregión.

En un mundo cada vez más globalizado, en el que desde hace varias décadas se marcha -aunque con coyunturales vaivenes- hacia la constitución de grandes bloques políticos, económicos, comerciales, e incluso militares, la integración sudamericana, más que una posibilidad o alternativa se ha convertido en una imperiosa necesidad.

Por tal motivo, ésta no puede ser vista como una empresa romántica que sólo busca rescatar el sueño de "la Patria Grande" de los Libertadores de América. Es mucho más que eso. Consolidar la integración de América del Sur es un elemento clave para que nuestros países no queden condenados a la irrelevancia económica y geopolítica en un cada vez más globalizado siglo XXI, así como para ofrecerles mayores y mejores posibilidades de bienestar $\mathrm{y}$ desarrollo a sus pueblos.

Integrarnos y convertirnos en un actor global, o quedar condenados a la irrelevancia como pequeños "países soberanos", sin ningún peso específico, sin importancia económica y sin ningún margen de maniobra en el juego de ajedrez del poder internacional. Esa es la disyuntiva que afrontamos los sudamericanos de este siglo y la respuesta, aunque compleja de implementar, resulta evidente: hay que avanzar decididamente hacia la integración subregional por una clara, sencilla y contundente razón, porque se- parados somos débiles pero juntos podemos ser muy fuertes ${ }^{9}$.

En esa línea, no hay que perder de vista el enorme potencial político y económico que tiene América del Sur en su conjunto. Así por ejemplo, en el ámbito económico los países sudamericanos alcanzaron en el año 2016 un producto interno bruto (PIB) de 5.6 billones de dólares, un ingreso per cápita promedio de 9,469.33 dólares y un volumen de exportaciones ascendente a 438,309 millones de dólares ${ }^{10}$, una cifra que triplica el volumen de exportaciones alcanzado en el año 2003.

Además, el vasto territorio que compone América del Sur, el mismo que representa el 12\% de la superficie terrestre, tiene al 2016 una población de 422,5 millones de personas según la CEPAL ${ }^{11}$, lo que equivale apenas al promedio del $6 \%$ de la población mundial. Un territorio con una muy baja densidad poblacional en comparación con otras áreas del planeta.

Adicionalmente, América del Sur y América Central en su conjunto, cuentan con el $4.13 \%$ de reservas de gas natural ${ }^{12}$ y el $19.4 \%$ de

9 Se cuenta que durante la Guerra de Las Malvinas en 1992, habiendo la Primera Ministra Británica Margaret Thatcher decidido enviar una expedición armada a las islas para recuperarlas tras la invasión argentina, uno de sus asesores dijo que quizás debía evaluarse mejor la reacción que podría tener América Latina frente a la incursión británica, a lo que la Sra. Thatcher respondió con desdén "América Latina no existe, sólo existen los países latinoamericanos".

10 Información extraída del portal especializado "Datosmacro.com".

11 Comisión Económica para América Latina y el Caribe, órgano de la Organización de las Naciones Unidas (ONU).

12 Fuente: el BP Statistical Review of World Energy 2013: 4.13\% de reservas mundiales de gas natural (equivalente a 270,9 Trillones de metros cúbicos). 
reservas de petróleo crudo en el mundo ${ }^{13}$, correspondiendo más del 80 por ciento de dichas reservas a los países sudamericanos. Y como si fuera poco, en tiempos de cambio climático y calentamiento global, los países sudamericanos cuentan con alrededor del 20\% del agua dulce de la Tierra, siendo la Amazonía el principal pulmón verde del planeta.

Desde el ámbito político, y pese a los graves retrocesos en estándares democráticos en países como Venezuela durante los últimos años, y escándalos de corrupción como el de la empresa constructora brasilera "Odebretch", el mismo que ha implicado a numerosos políticos y funcionarios públicos en América Latina, lo cierto es que la democracia representativa ha ido echando raíces progresivamente en nuestros países, por lo que periódicamente y desde hace casi tres décadas, se producen elecciones libres, transparentes y competitivas, así como la alternancia en el ejercicio del poder, con la lamentable excepción venezolana.

Además Sudamérica, aunque no exenta de sus problemas de inequidad social y presencia del crimen organizado como el narcotráfico, es esencialmente una región de paz, donde los conflictos existentes entre Estados se resuelven por medios pacíficos (diplomáticos y/o jurisdiccionales) $)^{14}$, donde no existe violencia y tensiones de orden religioso, étnico o racial como en tantos otros lugares del mundo. En este contexto, es una muy buena noticia para la región el Acuerdo de Paz alcanzado en Colombia entre el gobierno de ese país y la guerrilla

13 Fuente: el BP Statistical Review of World Energy 2015: 19.4\% de reservas mundiales de petróleo crudo.

14 El diferendo por la frontera marítima entre el Perú y Chile se resolvió el año 2014 a través de una sentencia de la Corte Internacional de Justicia (CIJ) de la Haya. Asimismo, se encuentra pendiente de solución ante la CIJ la demanda interpuesta por Bolivia contra Chile buscando una negociación efectiva de cara a solucionar el problema de mediterraneidad del Estado boliviano. de la $\mathrm{FARC}^{15}$, poniendo fin de este modo a 40 años de guerra interna.

Es por ello que, como bien señala el Ex Canciller de la República y ex Secretario General de la CAN, Embajador Allan Wagner Tizón, "en el contexto de la globalización, los países sudamericanos requieren adoptar estrategias para alcanzar una inserción competitiva e incluyente en nuevos y más exigentes escenarios internacionales...se trata de utilizar mejor aptitudes regionales, así como fortalecer capacidades de negociación y proyección internacionales. La Comunidad Sudamericana de Naciones es una herramienta para ello y la cohesión de la Comunidad Andina un instrumento poderoso para avanzar en estos propósitos"16.

III. Procesos de Integración en América del Sur: entre la ineficacia y la dispersión.-

Desde la segunda mitad del siglo XX hasta nuestros días, son múltiples los procesos de integración puestos en marcha en América Latina y el Caribe, desde procesos de promoción del libre comercio e integración económica, hasta procesos con un claro énfasis en la búsqueda de la integración política; y sin embargo, lo logrado y lo avanzado en todo este tiempo es a todos luces insatisfactorio en términos de resultados.

Sobre el particular, Francisco Rojas Aravena refiere que "a lo largo de la última década $y$ media, se establecieron y desarrollaron una serie de nuevas iniciativas tendientes a la formación de acuerdos de libre comercio, uniones

15 Fuerzas Armadas Revolucionarias de Colombia (FARC).

16 Wagner Tizón, Allan: "Comunidad Andina de Naciones: Integración para el Desarrollo en la Globalización”, en “Apertura a la Globalización. Desafíos y oportunidades en el Perú", Bernardo Haour S.J. Compilador, pag. 237. Fondo Editorial Universidad Antonio Ruiz de Montoya, Lima - 2007. 
aduaneras o sistemas de integración amplios, orientados hacia la conformación de comunidades comerciales-económicas y/o políticas subregionales. Estas renovadas iniciativas buscan superar procesos de integración de larga data, la mayoría provenientes de fines de la segunda post Guerra Mundial".

El resultado neto de este fenómeno, sin embargo, se expresa en un "exceso", una sobreoferta, de iniciativas y propuestas referidas a los procesos de integración que, no obstante su relevancia política y a pesar de ella, no han sido capaces de articular una visión más global de la región, y más bien la han fragmentado. Por eso es que hoy los acuerdos de integración no permiten hablar de una, sino de "varias" Américas Latinas"17.

En lo que América del Sur estrictamente se refiere, son cinco (5) los esquemas de integración vigentes que articulan a los doce países que lo conforman. Ello son en orden de antigüedad la Comunidad Andina - CAN (1969), la Asociación Latinoamericana de Integración - ALADI (1980), el Mercado Común del Sur - MERCOSUR (1991), la Unión de Naciones Suramericana - UNASUR (2004) y la Alianza del Pacífico (2011). De todos ellos, el que parece funcionar mejor y que ha despertado una gran expectativa en la comunidad internacional pese a su corta existencia es la Alianza de Pacífico. Los demás afrontan diversas crisis y cierta parálisis institucional.

Así por ejemplo, la CAN se debilitó tras la abrupta salida de Venezuela y su precipitada huida hacia el MERCOSUR en el 2006, luego que el Perú y Colombia anunciaran su interés de negociar y suscribir tratados de li-

17 Rojas Aravena, Francisco: "La Integración Regional: un proyecto Político-Estratégico", III Informe del Secretario General de FLACSO, p.p. 15-16, Facultad Latinoamericana de Ciencias Sociales (FLACSO)-Secretaría General, San José-Costa Rica, 2007. bre comercio con los Estados Unidos. Además, la CAN está sumida en una virtual parálisis al estar integrada por cuatro Estados que en la práctica componen dos bloques de países con ideas económicas y modelos de desarrollo distintos: el Perú y Colombia (además socios en la Alianza del Pacífico) por un lado, y Ecuador y Bolivia (miembros del ALBA-TCP ${ }^{18}$ ) por otro.

En el MERCOSUR, las tradicionales tensiones de la Argentina con Brasil y Uruguay que caracterizaron la época en el poder de los esposos Kirchner ${ }^{19}$, has sido sustituidas por las crisis generadas por la poca estabilizadora presencia de la Venezuela "chavista", país que no ha reconocido al nuevo Presidente del Brasil tras la destitución de Dilma Rousseff ${ }^{20}$, y se ha rehusado a completar el proceso de adaptación normativa al bloque, al punto tal que sus demás socios no sólo lo han relevado de asumir la Presidencia Pro Tempore que le correspondía, sino que además, lo han suspendido de su condición de miembro pleno, en atención a sus álgidos problemas políticos y económicos, pero sobre todo por la reiteradas violaciones del gobierno del Presidente Maduro a la Constitución y a los derechos humanos de gran par-

18 La Alternativa Bolivariana para las Américas-Tratado de Comercio de los Pueblos (ALBA-TCP), se constituyó en La Habana, Cuba, por acuerdo suscrito entre Venezuela y Cuba en diciembre del 2004. Posteriormente se adhirieron al ALBA países como Bolivia, Ecuador, Nicaragua, entre otros. El ALBA basa su accionar en los principios de solidaridad, complementariedad y cooperación entre los países de América Latina y el Caribe.

19 Los esposos Néstor Kirchner (2003-2007) y Cristina Fernández de Kirchner (2007-2011 y 20112015) gobernaron la República Argentina durante 12 años a través de tres períodos presidenciales consecutivos.

20 La Presidente Dilma Rousseff fue destituida por el Senado de Brasil el día 31 de agosto de 2016, declarada culpable del delito de responsabilidad en el maquillaje de las cuentas fiscales y la firma de decretos económicos sin aprobación del Congreso por 61 votos contra 20. El Vicepresidente Michel Tener asumió como presidente en su reemplazo. 
te del pueblo venezolano que se identifica con la oposición al régimen.

Esta situación ha llevado a varios analistas a pensar que es probable que en el corto plazo pueda darse una ruptura del bloque tal como lo conocemos en la actualidad. La crisis ha llegado a tal punto, que en un artículo publicado hace unos meses en el diario "El Clarín" de Buenos Aires, el ex Vicecanciller argentino Roberto García Moritán, señalaba que "el MERCOSUR está con fractura expuesta"21, como una forma de graficar la difícil actualidad que vive dicha organización.

Por su parte el UNASUR, cuyo antecedente directo fue la Comunidad Sudamericana de Naciones (CSN) creada en el Cuzco en el año 2004, nació con el objetivo -luego abandonadode convertirse en el marco institucional para la convergencia de la CAN y el MERCOSUR. En la actualidad, tal como ocurre en la CAN, la existencia de dos bloques de países con ideas opuestas tanto en lo económico como en lo político, afecta claramente la viabilidad y el desarrollo del más ambicioso proyecto de integración sudamericano ${ }^{22}$. Asimismo, los escándalos de corrupción política en el Brasil (el caso Lava Jato que involucra a grandes empresas como Petrobras, Odebrecht, etc.) han minado el liderazgo natural que el coloso sudamericano siempre tuvo en el UNASUR.

Mientras tanto, la ALADI, que desde su cambio de nombre en 1980 (antes era la Asociación Latinoamericana de Libre Comercio -ALALC) languidece entre sus extensas e intrascendentes reuniones de su sede en Montevideo.

21 Clarín, Buenos Aires, edición del 09 de agosto de 2016.

22 Uno de esos bloques está conformado por países que integran "la órbita chavista", es decir Venezuela, Bolivia, Ecuador y Surinam, todos ellos además miembros del ALBA-TCP.
Sólo la Alianza del Pacífico goza de un dinamismo distinto, el mismo que la ha convertido en el proceso de integración que mayor expectativa internacional despierta entre los procesos de integración existentes en esta parte del mundo. El problema es que sólo tres países sudamericanos participan de la misma (Colombia, Chile y Perú) ${ }^{23}$ y es mirada con desconfianza y hasta con cierto desprecio por países autodenominados "antiimperialistas" como Venezuela y Bolivia.

Vistas las cosas con realismo, ante esta multiplicidad de iniciativas de integración con más o menos los mismos protagonistas, con un sobre esfuerzo de acciones políticas y diplomáticas, con una dispersión de recursos económicos y humanos, y con una ineficiente y burocrática replicación de estructuras institucionales comunitarias más o menos similares, resulta claro que, poco o casi nada, avanzaremos hacia la consolidación de un vigoroso y eficaz proceso de integración regional sudamericano.

Sobre esta situación, coincidimos en lo esencial con el profesor Pablo Celi, quien indica que "si la desagregación de la región ha sido una constante que conspira contra la sostenibilidad de los procesos y las organizaciones integracionistas, es necesario trabajar la confluencia y complementariedad de los distintos, sobre la base de acciones compartidas y acciones coincidentes, a partir de una redefinición política de la relación entre los procesos nacionales, el regionalismo y la integración, con un concepto de región diversa, plural y solidaria; profundizando el relacionamiento Sur-Sur y la convergencia entre los diversos mecanismos de integración presentes en la región, para lo cual el UNASUR continúa siendo una posibilidad en ciernes que debe concluir su prolongada fase germinal"24.

23 El cuarto miembro de la Alianza del Pacífico es México.

24 Celi De la Torre, Pablo: "La fragilidad de la UNASUR en un pantano de conflictos" en la Revista Nue- 
Por todo lo expuesto, la exigencia de estos tiempos es avanzar con decisión hacia la implementación y el desarrollo de un proceso de integración regional sudamericano serio, pragmático y eficaz; ello implica ponerse a trabajar inmediatamente para hallar una fórmula jurídica-política-institucional, que permita dentro de un mediano plazo la convergencia de los procesos de integración preexistentes, los mismos que en muchos aspectos se superponen, compiten y/o se mediatizan entre sí. Asimismo, creemos que la UNASUR tendría que constituirse en el gran marco institucional de referencia para dicha convergencia.

En este punto, quizás los gobiernos de los países sudamericanos deberían pedir formalmente el apoyo y el asesoramiento técnico de instituciones como el SELA ${ }^{25}$ y/o la CEPAL, para el desarrollo indispensable de una propuesta de esquema de convergencia de los procesos de integración existentes en la subregión.

En línea con lo señalado, resulta esperanzador que en un saludable gesto de realismo político, quizás como una reacción ante el neo proteccionismo impulsado por el Presidente de los Estados Unidos, Donald Trump, los Cancilleres de los países integrantes del MERCOSUR y de la Alianza del Pacífico reunidos en Buenos Aires el pasado mes de mayo del presente en el marco del Foro Económico Mundial sobre América Latina, acordaron una hoja de ruta de seis puntos con el objetivo de avanzar hacia la complementación de ambos bloques, sobre la base de un compromiso que

va Sociedad: Democracia y Política en América Latina, Edición Digital, mayo 2017, http://nuso.org

25 El Sistema Económico Latinoamericano y del Caribe (SELA), es un organismo regional intergubernamental con sede en Caracas, Venezuela, creado el 17 de octubre de 1975 mediante el Convenio de Panamá. El SELA está integrado por 27 países de América Latina y el Caribe y está dirigido a promover un sistema de consulta y coordinación para concertar posiciones y estrategias comunes en América Latina y el Caribe. tenga como pilares la promoción del libre comercio y el multilateralismo. Sin duda, ello constituye una gran noticia para la subregión.

Con claridad de objetivos y una fuerte decisión política orientada hacia la convergencia de sus varios procesos de integración, no debe de estar muy lejos el día en que América del Sur sea un gran Mercado Común compuesto por una Zona de Libre Comercio y una Unión Aduanera; un gran espacio geográfico, económico y social donde circulen libremente los bienes, servicios, capitales y personas de los Estados sudamericanos; donde las personas de esta parte del mundo tengan el mismo DNI, pasaposrte y moneda; donde la subregión tenga más fuerza, influencia y poder de negociación en el contexto internacional; y los más importante, donde hayamos podido forjar un espacio común con más democracia, libertad, bienestar, igualdad de oportunidades, solidaridad e inclusión.

No cabe duda que dicho objetivo no va a ser fácil de alcanzar. En realidad se trata de una tarea dura y compleja qué va a tomar grandes dosis de tiempo, paciencia, constancia, imaginación, concesiones recíprocas y respeto mutuo. La tarea es muy ardua, sin embargo es posible. Hacerlo realidad sería el mejor legado para las futuras nuevas generaciones de sudamericanos.

\section{Breves Conclusiones.-}

Tras lo expuesto a lo largo de los párrafos precedentes, podemos arribar a las siguientes conclusiones:

1. En el mundo globalizado en que vivimos la integración sudamericana debe ser un objetivo político-estratégico de primer orden para todos los países que integran la subregión. De 
este modo se fortalecerá la influencia y el poder de negociación de América del Sur en el contexto internacional.

2. El escaso éxito de los procesos de integración en América Latina, y muy especialmente en América del Sur, se debe en gran parte a la dispersión de esfuerzos en ese sentido, lo que en la práctica se traduce en la existencia de diversos bloques u organizaciones que en muchos aspectos se superponen, compiten y/o se mediatizan entre sí.

3. La diversidad de iniciativas de integración en el ámbito sudamericano, ha supuesto un sobre esfuerzo de acciones políticas y diplomáticas; una dispersión de recursos económicos y humanos; y la existencia de una in- eficiente y burocrática replicación de estructuras institucionales comunitarias más o menos similares; lo que en términos prácticos no ha conducido a grandes logros en términos de integración regional.

4. En tal sentido, hay que trabajar con miras a concretar la convergencia de los distintos procesos de integración en América del Sur, teniendo a la UNASUR como marco institucional de referencia, dentro del cual puedan complementarse esquemas de integración como la CAN, el MERCOSUR y la Alianza del Pacífico.

5. Para el desarrollo de dicho plan de convergencia institucional, debería contarse con la asesoría y el apoyo técnico de entidades como la CEPAL y el SELA. 